\title{
On a Primal-Dual Analytic Center Cutting Plane Method for Variational Inequalities*
}

\author{
M. DENAULT $* *$ \\ J.-L. GOFFIN ${ }^{\dagger}$ \\ GERAD, McGill University, Montréal \\ Québec, Canada \\ Received October 8, 1997; Accepted August 11, 1998
}

jlg@crt.umontreal.ca

\begin{abstract}
We present an algorithm for variational inequalities $V I(\mathcal{F}, Y)$ that uses a primal-dual version of the Analytic Center Cutting Plane Method. The point-to-set mapping $\mathcal{F}$ is assumed to be monotone, or pseudomonotone. Each computation of a new analytic center requires at most four Newton iterations, in theory, and in practice one or sometimes two. Linear equalities that may be included in the definition of the set $Y$ are taken explicitly into account.

We report numerical experiments on several well-known variational inequality problems as well as on one where the functional results from the solution of large subproblems. The method is robust and competitive with algorithms which use the same information as this one.
\end{abstract}

Keywords: variational inequalities; analytic center, cutting plane method, monotone mappings, interior points methods, Newton's method, primal-dual.

Dedication To Olvi Mangasarian, who always was an inspiration, as a researcher and as a human being. His now classic black and blue book on nonlinear programming was the cleanest book available on this topic when I (JLG) was a student. Its high and uncompromising standards had much to do with my training in this field.

Olvi's smile and personality have made many a meeting a more enjoyable experience than expected.

\section{Introduction}

The finite dimensional variational inequality problem (VI) is a very significant extension to the problem of optimizing a nonlinear function, as it allows for the modelling of systems involving multiple agents, each pursuing his own objective, while sharing joint resources. The computation of economic equilibria, Nash equilibria and discretized free boundary problems is often performed using a formulation as variational inequalities.

The variational inequality problem is a generalization of the linear and nonlinear complementarity problems and of the mixed nonlinear complementarity problem — to which it can often be reduced, but at the cost of an increase in the number of variables.

Excellent references on variational inequalities are the monograph by Harker [16], the book by Nagurney [35], the review article of Harker and Pang [17], the short course of Marcotte [31], and the proceedings book of Ferris and Pang [9].

* Corresponding author: J.-L. Goffin, GERAD, École des Hautes Études Commerciales, 3000 chemin de la Côte-Sainte-Catherine, Montréal (Québec), Canada, H3T 2A7.

** Research supported in part by McGill University Fellowships

$\dagger$ Research supported by NSERC grant OPG0004152 and by the FCAR 
The development of algorithms for variational inequalities is an active and challenging research area. The variational inequality problem can be formulated as finding the zero of a generalized equation, as a fixed point problem or as a mixed nonlinear complementarity problem; these reformulations usually require additional assumptions.

The projection or fixed point methods may be viewed as extensions to VIs of subgradient optimization (Konnov [23]); more sophisticated versions include the extragradient method of Korpolevitch [24] and its variant by Solodov and Tseng [40]. Another class of approaches, pioneered by Mangasarian [29], in the case of nonlinear complementarity problems, uses a reformulation as a system of nonsmooth equations, which can also be viewed as a nonsmooth optimization problem; analogous smooth or nonsmooth reformulations can be found in the articles of Facchinei, Fischer, Kanzow [8] and Xiao, Harker [45, 46]. Optimization formulations involving the primal gap function, to which a proximal term is added, can be found in Fukushima [10], Kanzow, Qi [20] and Sun, Fukushima, Qi [42]. Ralph and Wright [38] describe an interior-point algorithm for VIs in a mixed nonlinear complementarity form.

Most of these algorithms need to make assumptions about the variational inequality, such as

1. the VI map is single-valued

2. it is differentiable, and the Jacobian can be used

3. the feasible set is given by a set of differentiable constraints.

Another approach, which extends nondifferentiable optimization to VIs, is to use a cutting plane description of the VI. This approach uses the fact that the VI functional separates the current point from the optimal set. Classical methods such as Kelley's cutting plane method, or by duality the Dantzig-Wolfe column generation, could be used. Lüthi [26] uses the ellipsoid method in this context, and Magnanti and Perakis [27] extend this approach to other centering methods. Lemaréchal, Nemirovskii, Nesterov [25] study the complexity of a level bundle method. The Analytic Center Cutting Plane (ACCPM), introduced in [11], uses the centering concept made familiar by interior point methods, i.e. the analytic center, in the context of column generation or cutting planes, and it has been shown, in practice, to be more effective than other centering schemes. The view of the VI as a convex feasibility problem was used by Goffin, Marcotte, Zhu [13] to describe and analyze a conceptual extension of ACCPM to the VI problem, using the results of [12]. A proximal analytic center cutting plane method, analyzed very nicely in a conic space, was introduced by Nesterov and Vial [37], and its complexity studied in the VI case.

The algorithm described here is a fully implementable version of [13], and we describe in detail a very effective method to compute the next analytic center from the previous one, using a primal-dual infeasible Newton method. The choice of an updating direction that involves a centering component leads, in practice, to a method that often needs no additional recentering Newton steps.

The algorithm applies to VIs defined by a monotone or pseudomonotone multivalued functional, possibly requiring the computation of subproblems, over a convex set $Y$ that could be defined by a separation oracle, and linear inequalities or equalities. These features are useful if the Jacobian is expensive or impossible to evaluate, or if it does not exist. 
The explicit accounting for linear equalities is useful for example in the case of network constraints.

As cutting plane methods do not necessarily generate a convergent sequence in the case of VIs, we introduce an auxiliary sequence of points, constructed by an averaging process, involving the primal variables computed by ACCPM, and which displays better convergence properties than the sequence of centers. Some intuitive justification can be found in Nesterov and Vial [37].

The article is organized as follows. We review in Section 2 some concepts of variational inequalities and analytic centers and we describe in Section 3 the algorithm in detail. In Section 4 we present some technical points concerning our MATLAB implementation of the method, and conclude in Section 5 with several numerical examples that range from typical economical equilibria to a pollution permits market model and to financial options pricing. The method is robust and competitive with algorithms which use the same information (mapping evaluations, but no Jacobian evaluations).

We use the following notation: $2^{\mathbf{R}^{m}}$ is the set of all subsets of $\mathbf{R}^{m}$; given a vector $x, X$ is the diagonal matrix with $x$ along the diagonal; $e$ is a vector of ones whose dimension can be inferred from the context.

\section{Basic Concepts}

In this section we give the definitions and present the basic concepts of variational inequalities and analytic centers.

\subsection{Variational Inequalities}

Definition 1 Let $\mathcal{F}$ be a point-to-set map from $\boldsymbol{R}^{m}$ into $2^{\boldsymbol{R}^{m}}$. Let $Y$ be a nonempty convex subset of $\boldsymbol{R}^{m}$. Then a solution to the variational inequality problem, denoted as $V I(\mathcal{F}, Y)$, is a point $y^{*} \in Y$ such that

$$
F\left(y^{*}\right)^{t}\left(y-y^{*}\right) \geq 0 \text { for some } F\left(y^{*}\right) \in \mathcal{F}\left(y^{*}\right) \text { and } \forall y \in Y .
$$

Geometrically, a solution $y^{*}$ is a point of $Y$ such that there exists a $F\left(y^{*}\right) \in \mathcal{F}\left(y^{*}\right)$ which makes an acute angle with all feasible directions, i.e. $-F\left(y^{*}\right)$ belongs to the normal cone to $Y$ at $y^{*}$, denoted as $N_{Y}\left(y^{*}\right)$ :

$$
-F\left(y^{*}\right) \in N_{Y}\left(y^{*}\right):=\left\{z:\left(y-y^{*}\right)^{t} z \leq 0 \forall y \in Y\right\} ;
$$

this can be written as:

$$
0 \in \mathcal{F}\left(y^{*}\right)+N_{Y}\left(y^{*}\right)
$$

which is the formulation as a generalized equation.

Definition 2 A point-to-set mapping $\mathcal{F}: \boldsymbol{R}^{m} \rightarrow 2^{\boldsymbol{R}^{m}}$ is monotone over a set $Y$ if

$$
\begin{gathered}
\left(F\left(y_{1}\right)-F\left(y_{2}\right)\right)^{t}\left(y_{1}-y_{2}\right) \geq 0 \\
\forall F\left(y_{1}\right) \in \mathcal{F}\left(y_{1}\right), \forall F\left(y_{2}\right) \in \mathcal{F}\left(y_{2}\right), \forall y_{1}, y_{2} \in Y .
\end{gathered}
$$


It is maximal monotone if the graph of $\mathcal{F}$, defined as

$$
G(\mathcal{F})=\{(y, t): y \in \operatorname{dom} Y, t \in \mathcal{F}(y)\}
$$

is maximal, i.e. for any monotone map $\mathcal{F}^{\prime}, G(\mathcal{F}) \subset G\left(\mathcal{F}^{\prime}\right)$ implies $G(\mathcal{F})=G\left(\mathcal{F}^{\prime}\right)$.

A common example of a maximal monotone mapping is the subdifferential of a convex function.

Definition 3 A point-to-set mapping $\mathcal{F}: \boldsymbol{R}^{m} \rightarrow 2^{\boldsymbol{R}^{m}}$ is pseudomonotone over a set $Y$ if

$$
\begin{gathered}
F\left(y_{2}\right)^{t}\left(y_{1}-y_{2}\right) \geq 0 \Rightarrow F\left(y_{1}\right)^{t}\left(y_{1}-y_{2}\right) \geq 0 \\
\forall F\left(y_{1}\right) \in \mathcal{F}\left(y_{1}\right), \forall F\left(y_{2}\right) \in \mathcal{F}\left(y_{2}\right), \forall y_{1}, y_{2} \in Y
\end{gathered}
$$

Definition 4 Let $\mathcal{F}$ be a point-to-set map from $\boldsymbol{R}^{m}$ into $2^{\boldsymbol{R}^{m}}$. Let $Y$ be a nonempty convex subset of $\boldsymbol{R}^{m}$. Then a weak solution to the variational inequality problem is a point $y^{*} \in Y$ such that

$$
F(y)^{t}\left(y-y^{*}\right) \geq 0 \quad \forall y \in Y \text { and } \forall F(y) \in \mathcal{F}(y) .
$$

The following theorem, from [36], relates weak solutions to solutions in the monotone case.

Theorem 1 Let $Y$ be a nonempty, closed, convex subset of $\boldsymbol{R}^{m}$, with nonempty interior, and let $\mathcal{F}$ be a monotone mapping with domain $\operatorname{dom}(\mathcal{F}), \operatorname{int}(Y) \subset \operatorname{dom}(\mathcal{F}) \subset Y$, then

1. Every solution of the variational inequality is also a weak solution;

2. Conversely, provided either:

- $Y \subset \operatorname{dom}(\mathcal{F})$ and $\mathcal{F}$ is single-valued continuous, or

- $\mathcal{F}$ is maximal monotone,

every weak solution solves the variational inequality.

In the case of pseudomonotone mappings, the following theorem was shown by Crouzeix [5] and Konnov [23]:

Theorem 2 Let $Y$ be a nonempty closed convex subset of $\boldsymbol{R}^{m}$ and let $\mathcal{F}$ be a pseudomonotone mapping from $Y$ into $2^{\boldsymbol{R}^{m}}$, with $\mathcal{F}(y)$ assumed to be a nonempty convex set for all $y \in Y$, and $\mathcal{F}$ assumed to be upper semi-continuous on $Y$.

Then every weak solution solves the variational inequality and conversely.

The key observation is that the solution set $Y^{*}$ can be described as the intersection of an infinite number of half-spaces:

$$
Y^{*}=\cap_{y \in Y} \cap_{F(y) \in \mathcal{F}(y)}\left\{y^{*} \in Y \mid F(y)^{t}\left(y-y^{*}\right) \geq 0\right\}
$$

This formulation is the basis of the cutting-plane approach to variational inequalities. 
It is thus possible to solve the $V I(\mathcal{F}, Y)$ as a convex feasibility problem, where $F(y)$ is the vector that separates $y$ from $Y^{*}$. A generic algorithm to find a point $y^{*} \in Y^{*}$ would be as follows:

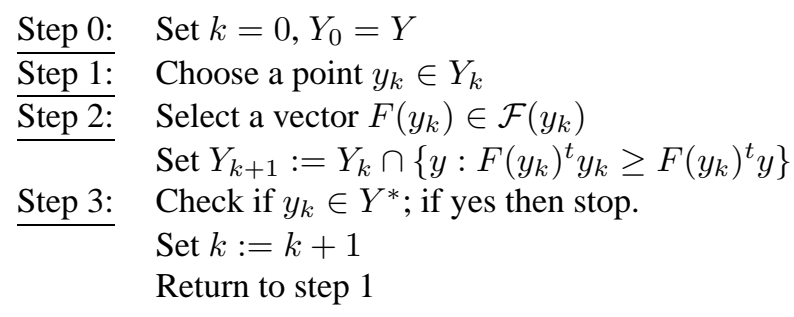

Note that the set $Y$ could be defined implicitly by a separation oracle, such that whenever $y_{k} \notin Y$, a feasibility cut separating $y_{k}$ from $Y$ is returned and added to the description of $Y_{k}$.

The selection of an appropriate stopping criterion is still an issue of some debate. We have used the primal gap function:

Definition 5 The primal gap function $g$ associated with $V I(\mathcal{F}, Y)$ is defined as

$$
g(y)=\min _{F(y) \in \mathcal{F}(y)} \min _{z \in Y} F(y)^{t}(z-y)
$$

In general $g(y) \leq 0$ and in particular $y^{*}$ is a solution of $V I(\mathcal{F}, Y)$ if and only if $g\left(y^{*}\right)=0$. If furthermore $Y$ is polyhedral and $\mathcal{F}$ single-valued, the gap can be evaluated by solving a linear optimization problem. An approximate solution can now be defined, in terms of the gap function:

Definition 6 For the variational inequality $V I(F, Y)$, the point $y^{*}$ is an $\epsilon$-approximate solution if $y^{*} \in Y$ and

$$
g\left(y^{*}\right) \geq-\epsilon
$$

If $\mathcal{F}$ is multivalued then a reasonable stopping criterion is:

$$
\min _{z \in Y} F\left(y^{*}\right)^{t}\left(z-y^{*}\right) \geq-\epsilon
$$

for the $F\left(y^{*}\right) \in \mathcal{F}\left(y^{*}\right)$ selected in step 2 above.

The following assumption is common to most cutting plane methods, and will be made for the remainder of this paper:

Assumption 1 The set $Y$ is assumed to be convex and compact. 


\subsection{Analytic Centers}

The analytic center of a polyhedron defined by a set of linear inequalities was introduced by G. Sonnevend [41]. An explicit treatment of linear equalities in $Y$ is introduced here, to avoid the traditional approach of rewriting unrestricted variables as the difference of two positive variables. Unrestricted variables arise through duality when equality contraints are present, and create instabilities in centering methods.

Consider the set

$$
Y=\left\{y \mid A^{t} y \leq c, B y=d\right\}
$$

and the associated dual potential function

$$
\varphi_{D}(y)=-\sum_{i=1}^{n} \ln \left(c_{i}-A_{i}^{t} y\right)=-\sum_{i=1}^{n} \ln \left(s_{i}\right)
$$

where $n$ is the number of components of $c$ and the number of rows of $A^{t}$, while $s=c-A^{t} y$ are the dual slacks. In addition to the boundedness of $Y$, the following assumption is required for analytic centers to be well defined.

Assumption 2 The set $Y$ satisfies a Slater condition, that is:

$$
\left\{y \mid A^{t} y<c, B y=d\right\}
$$

is not empty.

Definition 7 The analytic center $y^{c}$ of $Y$ is defined as the point minimizing the dual potential function over the set $\bar{Y}:=\left\{y \mid A^{t} y<c, B y=d\right\}$ :

$$
y^{c}=\arg \min _{y \in \bar{Y}} \varphi_{D}(y) .
$$

It is well known that, under the above assumptions, the dual potential function is strictly convex on $\left\{y \mid A^{t} y<c, B y=d\right\}$, and thus the center $y^{c}$ is unique. The necessary and sufficient first-order optimality conditions to:

$$
\begin{array}{ll}
\operatorname{minimize}_{y, s} & -\sum_{i=1}^{n} \ln s_{i} \\
\text { subject to } & A^{t} y+s=c \\
& B y=d \\
& s>0
\end{array}
$$

are thus:

$$
\begin{aligned}
A x+B^{t} \mu & =0 \\
A^{t} y+s & =c \\
B y & =d \\
X s & =e \\
x, s & >0
\end{aligned}
$$


where $x$ and $\mu$ are the variables associated with, respectively, the inequality and equality constraints. Equations (1) are referred to as the primal feasibility conditions, (2) and (3) are the dual feasibility conditions, and (4) the centrality conditions.

These optimality conditions are also those that minimize:

1. the primal potential function $\phi_{P}(x)=n \ln \left(c^{t} x+d^{t} \mu\right)-\sum_{i=1}^{n} \ln x_{i}$

2. the primal-dual potential fuction $\phi_{P D}(x, s)=n \ln x^{t} s-\sum_{i=1}^{n} \ln x_{i} s_{i}$; the minimum value of the primal- -dual potential function at the analytic center is the known value $n \ln n$. Note that for primal and dual feasible solutions $c^{t} x+d^{t} \mu=x^{t} s$ and thus $\phi_{P D}=\phi_{D}+\phi_{P}$.

An approximate analytic center is usually defined as a point $(y, x, s, \mu)$ which satisfies (1-3) and (5), and $\|e-X s\| \leq \eta<1$; the parameter $\eta$ is thus used to control the quality of the approximation. This proximity measure could be replaced by one involving the primal-dual potential function.

\section{An Analytic Center Cutting Plane Algorithm}

We now describe our algorithm. We make the assumption of a polyhedral set $Y$; as mentioned earlier, a general convex $Y$ can be treated by outer approximation (feasibility cuts).

Consider the problem $\operatorname{VI}(\mathcal{F}, Y)$ :

$$
\begin{aligned}
\text { Find } y^{*} \text { and } F\left(y^{*}\right) \in \mathcal{F}\left(y^{*}\right) & \text { such that } F\left(y^{*}\right)^{t}\left(y-y^{*}\right) \geq 0 \forall y \in Y \\
& \text { where } Y=\left\{y \mid A^{t} y \leq c, B y=d\right\}
\end{aligned}
$$

where $\mathcal{F}$ is a pseudomonotone or monotone mapping, $A$ is a $m \times n$ matrix and $B$ is a $p \times m$ matrix with $p<m$. We assume that $Y$ is bounded, possibly after the addition of a box $l \leq y \leq u$.

Then the following algorithm can be used to find an $\epsilon$-approximate solution of $\operatorname{VI}(\mathcal{F}, Y)$ :

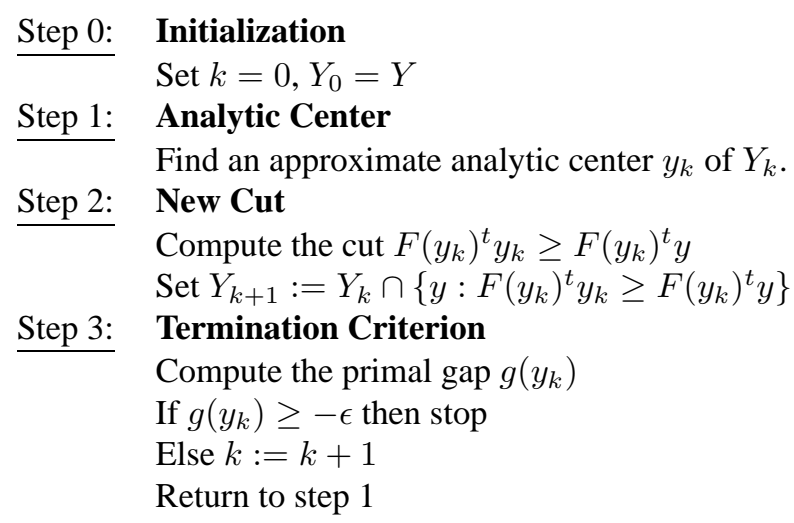

Step 1, computing an analytic center, for $k \geq 1$, is done with a primal-dual Newton method which uses the last center as a hot start. 
Step 2 consists basically of a function evaluation; the relative time spent on this step varies with the application, as will become clear in the section on numerical experiments.

Although the gap computation in Step 3 involves solving a linear program, this can usually be done very quickly, as the size of most VIs is small relative to the size of LPs that can be solved efficiently. Furthermore, it is not crucial to evaluate the gap at every iteration. If $\mathcal{F}\left(y_{k}\right)$ is multivalued, and thus in practice not explicitly available, the gap is replaced by $\min _{y \in Y}\left\{F\left(y_{k}\right)^{t}\left(y-y_{k}\right)\right\}$.

The rest of this section is devoted to a method for finding the approximate analytic center $y_{k+1}$ of $Y_{k+1}$, starting from $y_{k}$. The efficiency of this step is crucial to the whole algorithm.

\subsection{An Infeasible Primal-Dual Newton Step}

After $k$ iterations the set of localization is:

$$
Y_{k}=\left\{y \mid A_{k}^{t} y \leq c_{k}, B y=d\right\},
$$

where $A_{k}^{t} y \leq c_{k}$ includes both the $n$ initial inequality constraints and the $k$ generated inequalities (Step 2 of the algorithm). Let also $n_{k}=n+k$.

Assumption 3 We assume that the original matrices $A^{t}$ and $B$ have maximum rank.

Thus the current matrix $A_{k}^{t}$ has also maximum rank.

At iteration $k$, the approximate analytic center $y_{k} \in \mathbf{R}^{m}$ of $Y_{k}$ is a solution, together with $0<x_{k} \in \mathbf{R}^{n_{k}}, \mu_{k} \in \mathbf{R}^{p}$ and $0<s_{k} \in \mathbf{R}^{n_{k}}$, of the system of equations:

$$
\begin{aligned}
A_{k} x+B^{t} \mu & =0 \\
A_{k}^{t} y+s & =c_{k} \\
B y & =d
\end{aligned}
$$

while

$$
\delta:=e-X s \quad \text { with } \quad\|\delta\| \leq \eta<1
$$

The center $y_{k+1}$ of $Y_{k+1}$, the next set of localization, solves:

$$
\begin{aligned}
A_{k} x+a \xi+B^{t} \mu & =0 \\
A_{k}^{t} y+s & =c_{k} \\
a^{t} y+\sigma & =a^{t} y_{k} \\
B y & =d \\
X s & =e \\
\xi \sigma & =1 \\
x, s, \xi, \sigma & >0
\end{aligned}
$$

where $a:=F\left(y_{k}\right)$. Here $\xi$ and $\sigma$ are "new" unidimensional variables corresponding to the new constraint (8), and $n_{k+1}=n_{k}+1=n+k+1$.

The system (6)-(12) is solved iteratively with Newton's method; the initial point we use is $y=y_{k}, x=x_{k}, s=s_{k}$ while the new variables $\sigma$ and $\xi$ are given positive values that 
must be carefully chosen to achieve the recovery of primal and dual feasibility and make the centering as efficient as possible. For values of $\xi=0$ and $\sigma=0$ this initial point is feasible in the sense of (6)-(9), although it will not be feasible with respect to strict positivity. For positive initial values of $\xi$ and $\sigma$, which are required for interior point methods, the solution is infeasible to both (6) and (8). A full Newton step is needed to recover the feasibility conditions (6), (7) and (8).

The choice of a primal-dual method is motivated by the observation that it appears to be the most efficient interior point method available.

To improve the clarity of the notation, we now drop the index $k$, and replace it by a bar, so that the current center of $\bar{Y}:=Y_{k}$ is denoted as $(\bar{y}, \bar{s}>0, \bar{\mu}, \bar{x}>0)$, and $\bar{A}:=A_{k}$ and $\bar{c}:=c_{k}$. The strictly positive values chosen for the new variables are denoted as $\bar{\xi}$ and $\bar{\sigma}$.

The Newton steps $d y, d x, d s, d \xi$ and $d \sigma$ from the initial point are given by the system:

$$
\begin{aligned}
\bar{A} d x+a d \xi+B^{t} d \mu & =-\bar{A} \bar{x}-a \bar{\xi}-B^{t} \bar{\mu}=-a \bar{\xi} \\
\bar{A}^{t} d y+d s & =-\bar{A}^{t} \bar{y}-\bar{s}+\bar{c}=0 \\
a^{t} d y+d \sigma & =-a^{t} \bar{y}-\bar{\sigma}+a^{t} \bar{y}=-\bar{\sigma} \\
B d y & =-B \bar{y}+d=0 \\
\bar{S} d x+\bar{X} d s & =e-\bar{X} \bar{s}=: \delta \\
\bar{\sigma} d \xi+\bar{\xi} d \sigma & =1-\bar{\xi} \bar{\sigma}
\end{aligned}
$$

From (14) and (17),

$$
\begin{aligned}
d s & =-\bar{A}^{t} d y \\
d x & =\bar{S}^{-1}(\delta-\bar{X} d s) \\
& =\bar{S}^{-1}\left(\delta+\bar{X} \bar{A}^{t} d y\right)
\end{aligned}
$$

Using (13) and the above we have

$$
\bar{A} d x=\bar{A} \bar{S}^{-1}\left(\delta+\bar{X} \bar{A}^{t} d y\right)=-B^{t} d \mu-a(\bar{\xi}+d \xi)
$$

from which, defining $\Delta:=\bar{A} \bar{S}^{-1} \bar{X} \bar{A}^{t}$, Dikin's matrix, and $\xi_{N}:=\bar{\xi}+d \xi, \sigma_{N}:=\bar{\sigma}+d \sigma$, the values of the new variables after a full Newton step, we have:

$$
d y=\Delta^{-1}\left(-\bar{A} \bar{S}^{-1} \delta-B^{t} d \mu-\xi_{N} a\right) .
$$

Combining (16) and the definition $H:=B \Delta^{-1} B^{t}$, we obtain

$$
B d y=-B \Delta^{-1} B^{t} d \mu-B \Delta^{-1}\left(\bar{A} \bar{S}^{-1} \delta+\xi_{N} a\right)=0
$$

and

$$
d \mu=-H^{-1} B \Delta^{-1}\left(\bar{A} \bar{S}^{-1} \delta+\xi_{N} a\right)
$$

We also use the notation

$$
P:=I-\Delta^{-1 / 2} B^{t} H^{-1} B \Delta^{-1 / 2}
$$




$$
\bar{r}^{2}:=a^{t} \Delta^{-1} a-a^{t} \Delta^{-1} B^{t} H^{-1} B \Delta^{-1} a=a^{t} \Delta^{-1 / 2} P \Delta^{-1 / 2} a
$$

where $P$ is a projection matrix. Note that $\Delta^{-1 / 2}$ does not need to be computed; a $L L^{t}$ Cholesky factorization of $\Delta$ is all that is needed in the computations. Clearly $a^{t} \Delta^{-1 / 2} P \Delta^{-1 / 2} a=$ $\left\|P \Delta^{-1 / 2} a\right\|^{2}$ is nonnegative. The following lemma shows that $\bar{r}=0$ if and only if the current iterate $\bar{y}$ is a solution of the VI.

Lemma 1 If $\bar{r}=0$, then $\bar{y}$ is a solution to the variational inequality.

\section{Proof:}

$\bar{r}=0$ implies that $P \Delta^{-1 / 2} a=0$. Or $\Delta^{-1 / 2} a=\Delta^{-1 / 2} B^{t} H^{-1} B \Delta^{-1} a$.

Hence: $a=B^{t} H^{-1} B \Delta^{-1} a$, i.e. $a \in \operatorname{Range}\left(B^{t}\right)$.

But as $\bar{s}>0, A^{t} \bar{y}<c$, and thus Range $\left(B^{t}\right)$ is both the normal cone of $Y$ at $\bar{y}$ and its opposite.

Q.E.D.

The expressions for $d y$ and $d \mu$ above and (15) yield

$$
\begin{aligned}
-\sigma_{N}=a^{t} d y= & -a^{t} \Delta^{-1}\left(\bar{A} \bar{S}^{-1} \delta+B^{t} d \mu+\xi_{N} a\right) \\
= & a^{t} \Delta^{-1} B^{t} H^{-1} B \Delta^{-1} a \xi_{N}-a^{t} \Delta^{-1} a \xi_{N}-a^{t} \Delta^{-1} \bar{A} \bar{S}^{-1} \delta \\
& +a^{t} \Delta^{-1} B^{t} H^{-1} B \Delta^{-1} \bar{A} \bar{S}^{-1} \delta \\
= & -\bar{r}^{2} \xi_{N}-a^{t} \Delta^{-1}\left(I-B^{t} H^{-1} B \Delta^{-1}\right) \bar{A} \bar{S}^{-1} \delta
\end{aligned}
$$

Introducing the notation

$$
\omega:=a^{t} \Delta^{-1}\left(I-B^{t} H^{-1} B \Delta^{-1}\right) \bar{A} \bar{S}^{-1} \delta=a^{t} \Delta^{-1 / 2} P \Delta^{-1 / 2} \bar{A} \bar{S}^{-1} \delta,
$$

we can then write

$$
-\bar{r}^{2} \xi_{N}+\sigma_{N}=\omega
$$

If we also rewrite (18) as

$$
\bar{\sigma} \xi_{N}+\bar{\xi} \sigma_{N}=1+\bar{\xi} \bar{\sigma}
$$

we obtain the values for $\xi_{N}$ and $\sigma_{N}$ :

$$
\begin{aligned}
\xi_{N} & =\frac{\bar{\xi}+\bar{\sigma}^{-1}-\bar{\xi} \bar{\sigma}^{-1} \omega}{1+\bar{\sigma}^{-1} \bar{\xi} \bar{r}^{2}} \\
\sigma_{N} & =\bar{r}^{2} \xi_{N}+\omega .
\end{aligned}
$$

Replacing $d \mu$ in the expression for $d y$, and $d y$ in the expressions for $d x$ and $d s$ one gets:

$$
\begin{aligned}
d y & =-\Delta^{-1 / 2} P \Delta^{-1 / 2} a \xi_{N}-\Delta^{-1 / 2} P \Delta^{-1 / 2} \bar{A} \bar{S}^{-1} \delta \\
d s & =\bar{A}^{t} \Delta^{-1 / 2} P \Delta^{-1 / 2} a \xi_{N}+\bar{A}^{t} \Delta^{-1 / 2} P \Delta^{-1 / 2} \bar{A} \bar{S}^{-1} \delta \\
d x & =\bar{S}^{-1}(\delta-\bar{X} d s) .
\end{aligned}
$$

To complete the analysis, let:

$$
K:=\bar{S}^{-1 / 2} \bar{X}^{1 / 2} \bar{A}^{t} \Delta^{-1 / 2} P
$$

it can be checked that: 
- $K^{t} K=P^{2}=P$

- $K K^{t}=K K^{t} K K^{t}$, and thus $K K^{t}$ is also a projection matrix, and we let $P_{2}=$ $I-K K^{t} ; K K^{t}$ is an oblique projection on the null space of $d s$.

Defining

$$
\begin{aligned}
p_{x} & :=P_{2} \bar{X}^{-1 / 2} \bar{S}^{-1 / 2} \delta \\
p_{s} & :=\left(I-P_{2}\right) \bar{X}^{-1 / 2} \bar{S}^{-1 / 2} \delta \\
\bar{p} & :=K \Delta^{-1 / 2} a,
\end{aligned}
$$

the Newton iterate for the old variables is then:

$$
\begin{aligned}
x_{N} & =\bar{X}\left(e+\bar{X}^{-1 / 2} \bar{S}^{-1 / 2}\left(p_{x}-\bar{p} \xi_{N}\right)\right) \\
s_{N} & =\bar{S}\left(e+\bar{X}^{-1 / 2} \bar{S}^{-1 / 2}\left(p_{s}+\bar{p} \xi_{N}\right)\right) \\
y_{N} & =\bar{y}-\Delta^{-1 / 2} P \Delta^{-1 / 2} a \xi_{N}-\Delta^{-1 / 2} P \Delta^{-1 / 2} \bar{A} \bar{S}^{-1} \delta \\
\mu_{N} & =\bar{\mu}-H^{-1} B \Delta^{-1}\left(\bar{A} \bar{S}^{-1} \delta+\xi_{N} a\right) ;
\end{aligned}
$$

The expression for $y_{N}$ is composed of a centering term and an updating one:

$$
-\Delta^{-1 / 2} P \Delta^{-1 / 2} a \xi_{N}
$$

this is a projection, in the metric given by Dikin's ellipsoid, of the functional $F(\bar{y})$ on the space of linear equality constraints. Thus the method may be viewed as a variable metric subgradient method, with a natural step-size (or trust region) given by Dikin's ellipsoid; this is the key reason for the efficiency of ACCPM. The scaled Newton direction for $x$ and $s$ is the sum of centering $\left(p_{x}, p_{s}\right)$ components and an updating $(\bar{p})$ component.

The initial values $\bar{\xi}$ and $\bar{\sigma}$ to be given to the new variables $\xi$ and $\sigma$ are critical to the efficiency of a primal-dual infeasible Newton method. Four options are discussed below; for two of them, we give results on the number of Newton steps needed to recenter.

- Option 1 We note that the central cut analysis of [14] applies directly to our problem, even if [14] did not involve dual equalities $B y=d$, and thus the algebra is different. That is, the following results hold:

1. $\bar{p}^{T} p_{x}=0$

2. $\|\bar{p}\|=\bar{r}$

3. $p_{x}^{t} p_{s}=0$

4. $p_{x}+p_{s}=\bar{X}^{-1 / 2} \bar{S}^{-1 / 2} \delta$

5. $\left\|p_{x} \circ p_{s}\right\| \leq \frac{\sqrt{2}}{4} \frac{\eta^{2}}{1-\eta}$ : this is the key lemma on the convergence of the primal-dual method, due to Mizuno, see [34]

6. $\left\|p_{x}\right\| \leq \frac{\eta}{\sqrt{1-\eta}}$ and $\left\|p_{s}\right\| \leq \frac{\eta}{\sqrt{1-\eta}}$

7. $|\omega|=\left|\bar{p}^{T} p_{s}\right| \leq\|\bar{p}\|\left\|p_{s}\right\| \leq \bar{r}\left\|\bar{X}^{-1 / 2} \bar{S}^{-1 / 2} \delta\right\| \leq \bar{r} \frac{\eta}{\sqrt{1-\eta}}$, 
where $\circ$ denotes the Hadamard or componentwise product. Denoting $\bar{\omega}:=\omega / \bar{r}, x^{+}:=$ $\left[x_{N} ; \xi_{N}\right]$ and $s^{+}:=\left[s_{N} ; \sigma_{N}\right]$, the following theorem can be proved (see [14]):

Theorem 3 If the infeasibilities are chosen as

$$
\bar{\xi}=\bar{\sigma}^{-1}=\frac{1-\sqrt{1-\beta(\beta+\bar{\omega})}}{\bar{r}(\beta+\bar{\omega})},
$$

then

$$
\begin{gathered}
\bar{r} \xi_{N}=\beta, \\
\sigma_{N} / \bar{r}=\beta+\bar{\omega},
\end{gathered}
$$

and

$$
\left\|X^{+} s^{+}-e\right\| \leq\left(\beta^{4}+(1-\beta(\beta+\bar{\omega}))^{2}\right)^{1 / 2}+\frac{\sqrt{2}}{4} \frac{\eta^{2}}{1-\eta}+\beta \frac{\eta}{\sqrt{1-\eta}} .
$$

Thusfor $\eta=.04$ and $\beta=1 / \sqrt{2}, x^{+}$and $s^{+}$are primal and dual feasible, and approximately centered, i.e.:

$$
\left\|X^{+} s^{+}-e\right\| \leq .757
$$

and thus 3 centering steps ( 1 dual, 2 primal-dual $)^{1}$ will suffice to recenter within $\eta=.04$.

Experimental results indicate clearly that the very strong centering $(\eta=.04)$ required in the proof of the above theorem is not best in practice. By loosening the recentering criterion (say to $\eta=.9$ or $\eta=.95$ ), centering steps are often not needed at all.

If a loose centering criterion is used it is clearly not possible to guarantee the approximate centering of the next iterate $\left(x^{+}, s^{+}\right)$; this can be managed as the primal- dual (or the dual or the primal) potential function can be used to guide the algorithm to the next center. However, in a primal-dual method, the strict positivity of $x^{+}, s^{+}$is absolutely essential (as the strict positivity of $x^{+}$or the strict positivity of $s^{+}$are required for a primal or a dual method, respectively).

The conditions for the positivity of the next iterate are:

1. $x_{N}>0$ if and only if $\bar{p}_{j} \xi_{N}<\left(\bar{x}_{j} \bar{s}_{j}\right)^{1 / 2}+\left(p_{x}\right)_{j} \forall j$

2. $s_{N}>0$ if and only if $-\bar{p}_{j} \xi_{N}<\left(\bar{x}_{j} \bar{s}_{j}\right)^{1 / 2}+\left(p_{s}\right)_{j} \forall j$

3. $\sigma_{N}>0$ if and only if $\bar{r}^{2} \xi_{N}>-\omega$.

4. $\xi_{N}>0$.

These four conditions amount to a ratio test that may not have, if $\eta$ is too large, any positive solution in $\xi_{N}$. Note that, from item 3, $\omega<0$ makes matters more difficult than $\omega \geq 0$. In fact it is easy to see that $\eta<1 / 3$ implies that the ratio test will be successful, but this may lead, at least in theory, to poor centering of $\left(x^{+}, s^{+}\right)$. This statement follows (see [14]) as: $x_{N}>0$ and $s_{N}>0$ is implied by $\bar{r} \xi_{N} \leq \frac{1-2 \eta}{\sqrt{1-\eta}}$, and $|\bar{\omega}| \leq \frac{\eta}{\sqrt{1-\eta}}$, so that a feasible value of $\bar{r} \xi_{N}$ exists if

$$
\frac{\eta}{\sqrt{1-\eta}}<\frac{1-2 \eta}{\sqrt{1-\eta}}
$$


that is $\eta<1 / 3$.

- Option 2 Another option would be to use instead of the linearized centering equation corresponding to the new variables

$$
\bar{\sigma} d \xi+\bar{\xi} d \sigma=1-\bar{\xi} \bar{\sigma}
$$

the actual quadratic relation

$$
(\bar{\xi}+d \xi)(\bar{\sigma}+d \sigma)=1
$$

which can be written as

$$
\xi_{N} \sigma_{N}=1 .
$$

This amounts to enforcing the exact centering for the new variables. This also maximizes the values of $\sigma_{N}$, and thus of $\xi_{N}$ in (19) and (20). This is achieved if $\bar{r} \bar{\xi}=\bar{r} / \bar{\sigma}=\frac{\sqrt{\bar{\omega}^{2}+4}-\bar{\omega}}{2}$, leading to $\bar{r} \xi_{N}=\frac{\sqrt{\bar{\omega}^{2}+4}-\bar{\omega}}{2}$ and $\sigma_{N} / \bar{r}=\frac{\sqrt{\bar{\omega}^{2}+4}+\bar{\omega}}{2}$, which are both positive for any value of $\bar{\omega}$. This can also be seen as setting $\beta=\frac{\sqrt{\bar{\omega}^{2}+4}-\bar{\omega}}{2}$ in Theorem 3 .

This option cannot guarantee that $\left(x_{N}, s_{N}, \xi_{N}^{2}, \sigma_{N}\right)>0$, as it is identical to option 1 if a linesearch on $\xi_{N}$ is used. In practice however, both seem to work, and this option seems more effective than option 1 , if no linesearch is used.

- Option 3 If the above fails, then an alternative approach would be to assume in equation (17) that $\delta=0$, thus ignoring the centering component of the Newton updating step; this is a pure predictor step, which ignores the centering component of the previous options. The formulas derived above are all still valid, provided one sets $\omega=0$ and $p_{x}=p_{s}=0$.

The ratio test then becomes:

1. $x_{N}>0$ if and only if $\bar{p}_{j} \xi_{N}<\left(\bar{x}_{j} \bar{s}_{j}\right)^{1 / 2}$

2. $s_{N}>0$ if and only if $-\bar{p}_{j} \xi_{N}<\left(\bar{x}_{j} \bar{s}_{j}\right)^{1 / 2}$

3. $\xi_{N}>0$, which implies $\sigma_{N}>0$,

which always has a positive solution in $\xi_{N}$. A sufficient condition for this ratio test to pass is that $\bar{\xi}$ and $\bar{\sigma}$ be set as in the theorem below, with the parameter $\beta$ satisfying $\beta<\sqrt{1-\eta}$.

Theorem 4 If the infeasibilities are chosen as

$$
\bar{\xi}=\bar{\sigma}^{-1}=\frac{\beta}{\bar{r}\left(1+\sqrt{1-\beta^{2}}\right)}=\frac{1-\sqrt{1-\beta^{2}}}{\bar{r} \beta},
$$

then

$$
\begin{gathered}
\bar{r} \xi_{N}=\beta, \\
\sigma_{N} / \bar{r}=\beta,
\end{gathered}
$$

and,

$$
\left\|X^{+} s^{+}-e\right\| \leq\left(\beta^{4}+\left(1-\beta^{2}\right)^{2}\right)^{1 / 2}+\eta .
$$


Thusfor $\eta=.08$ and $\beta=1 / \sqrt{2}, x^{+}$and $s^{+}$are primal and dualfeasible, and approximately centered. i.e.:

$$
\left\|X^{+} s^{+}-e\right\| \leq .7872
$$

and thus 3 centering steps ( 1 dual, 2 primal-dual) will suffice to recenter within $\eta=.08$.

Proof: The primal-dual proximity measure after the updating step is given by, denoting $\bar{p} \circ \bar{p}$ by $\bar{p}^{2}$,

$$
\begin{aligned}
\left(X^{+} s^{+}-e\right) & =\left(\begin{array}{c}
X_{N} s_{N}-e \\
\xi_{N} \sigma_{N}-1
\end{array}\right) \\
& =\left(\begin{array}{c}
-\bar{p}^{2} \xi_{N}^{2} \\
\xi_{N} \sigma_{N}-1
\end{array}\right)+\left(\begin{array}{c}
-\delta \\
0
\end{array}\right) .
\end{aligned}
$$

But we have

$$
\left\|\bar{p}^{2}\right\|^{1 / 2} \leq\|\bar{p}\|=\bar{r}
$$

Hence

$$
\begin{aligned}
\left\|X^{+} s^{+}-e\right\| & \leq\|\delta\|+\sqrt{\left(\left\|\bar{p}^{2}\right\| \xi_{N}^{2}\right)^{2}+\left(1-\sigma_{N} \xi_{N}\right)^{2}} \\
& \leq \eta+\sqrt{\left(\bar{r} \xi_{N}\right)^{4}+\left(1-\sigma_{N} \xi_{N}\right)^{2}}
\end{aligned}
$$

and the theorem follows.

In this case if $\eta$ is chosen rather loosely, say 0.9 , a value of $\beta<\sqrt{1-\eta}$ can be viewed as giving an initial value $\beta / \bar{r}$ to $\xi_{N}$, and then a linesearch can be used that minimizes the primal-dual potential.

- Option 4 A fourth approach could consist in using $\alpha \delta$, with $0<\alpha<1$, in equation (17), thus limiting but not ignoring the centering component of the Newton updating step. We did not test this last option in practice.

\subsection{Centering Newton Steps}

Once a primal and dual feasible point has been computed, Newton's method is used for computing an approximate analytic center of the updated set $Y_{k+1}$. ACCPM [11] used the primal projective method due to Karmarkar. One could also use a dual Newton method or a primal-dual Newton method. We describe the primal-dual Newton method first.

Let the bar notation now account for the $(k+1)^{t h}$ generated cut $(8), \bar{A}:=A_{k+1}$ and $\bar{c}:=c_{k+1}$. Then the new analytic center must solve

$$
\begin{aligned}
\bar{A} x+B^{t} \mu & =0 \\
\bar{A}^{t} y+s & =\bar{c} \\
B y & =d \\
X s & =e \\
x, s & >0 .
\end{aligned}
$$


After the update Newton step of section 3.1, the current point $(\bar{y}, \bar{s}, \bar{x}, \bar{\mu})$ is assumed to satisfy:

$$
\begin{aligned}
\bar{A} \bar{x}+B^{t} \bar{\mu} & =0 \\
\bar{A}^{t} \bar{y}+\bar{s} & =\bar{c} \\
B \bar{y} & =d \\
\|\bar{X} \bar{s}-e\| & \leq \eta<1 \\
\bar{x}, \bar{s} & >0 .
\end{aligned}
$$

The Newton iterate $\left(y^{+}, s^{+}, x^{+}, \mu^{+}\right)$can be computed exactly as in the previous section, just dropping the updating component, and thus:

$$
\begin{aligned}
x^{+} & =\bar{X}\left(e+\bar{X}^{-1 / 2} \bar{S}^{-1 / 2} p_{x}\right) \\
s^{+} & =\bar{S}\left(e+\bar{X}^{-1 / 2} \bar{S}^{-1 / 2} p_{s}\right) \\
y^{+} & =\bar{y}-\Delta^{-1 / 2} P \Delta^{-1 / 2} \bar{A} \bar{S}^{-1} \delta \\
\mu^{+} & =\bar{\mu}-H^{-1} B \Delta^{-1} \bar{A} \bar{S}^{-1} \delta
\end{aligned}
$$

Mizuno’s [34] Lemma 1 applies and:

$$
\left\|X^{+} s^{+}-e\right\|=p_{x} \circ p_{s} \leq \frac{\sqrt{2}}{4} \frac{\eta^{2}}{1-\eta}
$$

This shows that the recentering steps will improve $\left\|X^{+} s^{+}-e\right\|$ quadratically as long as $\eta<\frac{4}{4+\sqrt{2}}<0.739$, since this implies $\frac{\sqrt{2}}{4} \frac{\eta^{2}}{1-\eta}<\eta$.

On the other hand, a dual Newton method would take a Newton step for the dual potential, constrained by $\bar{A}^{t} y+s=\bar{c}, B y=d$, starting at $(\bar{y}, \bar{s})$. This gives the next dual iterate $\left(y^{+}, s^{+}\right)$. The primal iterate $\left(x^{+}, \mu^{+}\right)$is then computed by

$$
\min _{(x, \mu)}\left\{\left\|S^{+} x-e\right\|: A x+B^{t} \mu=0\right\}
$$

the formulas are not given here, but are similar to the ones given above, with $\bar{X}$ replaced by $\bar{S}^{-1}$, and thus using a different factorization. The standard quadratic convergence lemma is then:

$$
\left\|X^{+} s^{+}-e\right\| \leq \eta^{2} .
$$

If $\frac{4-\sqrt{2}}{4} \leq \eta<1$, a dual recentering can be used, as it is, in theory, stronger than a primaldual centering. If $\eta>1$ then a linesearch involving the primal-dual potential along the primal-dual Newton direction should be used; see the book by Ye [47, pp. 102-106] for details.

\subsection{Convergence and Averaging}

The convergence proofs of centering schemes that do not include a regularization term are very delicate. Lüthi [26] and Magnanti and Perakis [27] prove for a variety of volume reduction schemes that the volume of $Y_{k}$ converges to zero; convergence and complexity 
results require that $\mathcal{F}$ be single-valued and strongly monotone or f-monotone (this is also known as co-coercive).

The analytic center cutting plane method is not a volume reduction algorithm, and the convergence proof shows that the size of the largest sphere included in $Y_{k}$ converges to zero [12], and this implies convergence and complexity only under additional assumptions on $\mathcal{F}$.

Convergence and complexity were shown in [13] under the assumption that $\mathcal{F}$ is singlevalued pseudo-co-coercive ${ }^{2}$. The following theorem extends the results of [13] to the case involving linear equalities.

Theorem 5 The cutting plane algorithm which uses approximate analytic centers, and the primal-dual updating scheme, constructs a set $Y_{k}$ that does not contain a sphere of radius $\bar{\epsilon}$ after $O^{*}\left(\frac{(m-p)^{2}}{\bar{\epsilon}^{2}}\right)$ iterations. ${ }^{3}$ Under the additional condition on $\mathcal{F}$ that it is singlevalued and pseudo-co-coercive, the algorithm terminates with a primal gap $g\left(y_{k}\right) \geq-\epsilon$ in $O^{*}\left(\frac{(m-p)^{2}}{\epsilon^{2}}\right)$ iterations. ${ }^{4}$

The sequence of analytic centers $\left\{y_{0}, y_{1}, y_{2}, \ldots, y_{k}, \ldots\right\}$ produced by cutting plane methods does not need to converge to a solution without these strong assumptions. Nesterov and Vial [37] exhibit a two-dimensional monotone variational inequality for which the sequence of analytic centers converges to a point that is not a solution. They also show, for the proximal analytic center cutting plane method, that a sequence of certain convex combinations of previous centers will converge to a solution for any monotone VI problem.

Drawing an analogy between the Nesterov-Vial algorithm and ours, we construct, corresponding to each analytic center $y_{k}$, a point $\tilde{y}_{k}$ that is a convex combination of the $k$ previous analytic centers (recall that the initial analytic center is labeled $y_{0}$ ). The weights of the combination that we use are the last $k$ elements of the primal variable $x$, which are approximately the inverses of the slacks for the cuts generated at each of the previous centers. These weights are normalized by their sum; $\tilde{y}_{k}$ is then:

$$
\tilde{y}_{k}=\sum_{i=1}^{k} \frac{x_{(n+i)}}{\sum_{j=1}^{k} x_{(n+j)}} y_{i}
$$

Here $x_{(n+j)}$ refers to the $(n+j)^{\text {th }}$ component of $x_{k}$; also, recall that $n$ is the number of initial linear inequality constraints.

The sequence given by the $\tilde{y}_{k}$ points converges in practice to the solution for the NesterovVial problem. Also, the speed of convergence improves significantly for all problems tested. A full explanation and proof of the behaviour of this sequence is not yet available; but this averaging approach is traditional in many algorithms for fixed point problems and VIs ( [27], [28]).

Note that if the gap is computed at each iteration, two function evaluations are necessary per iteration: $F\left(y_{k}\right)$ to generate the cut and $F\left(\tilde{y}_{k}\right)$ to obtain the gap value. In step 3 of the algorithm in section 3 , the evaluation of the gap $g\left(\tilde{y}_{k}\right)$ thus replaces the evaluation of the gap $g\left(y_{k}\right)$. 


\section{Implementation of the algorithm}

The algorithm was implemented using the MATLAB language and environment, version 5.1. MATLAB is a convivial setting for matrix computations and algorithm development. With the computing intensive tasks devoted to compiled subroutines, it can also be competitive with C or Fortran codes, see for example the LIPSOL solver of Zhang [48].

- Factorization The update Newton step described in Section 3.1 implies the solution of a linear system, as well as the solution of a second smaller system when equality constraints are present. The same thing is true for the recentering Newton step of Section 3.2.

The solution of linear systems is performed by dense Cholesky factorizations. Because of the density of the introduced cuts, the matrices to be factored are dense; however, they have the dimension $m \times m$ of the problem's size, independent of the potentially large number of cuts generated.

When equality constraints are present, one additional $p \times p$ matrix is factored at each step. We apply, when necessary, the simple technique of overweighing the diagonal elements if the matrix appears to become singular.

- Sparsity and Products The sparsity of the initial constraints, box and others, is exploited whenever possible in the matrix products. This is made easy by MATLAB's sparsity handling capability.

- Initial center An initial analytic center is needed to start the algorithm. When only box constraints are involved, this is a trivial task. When more complex constraints are present, the recentering subroutine is applied to a point that is feasible by construction.

- Stopping criterion The gap function $g(y)$ is used as a stopping criterion. Its evaluation at the point $\tilde{y}_{k}$ (see (27)) corresponds to solving the linear optimization problem

$$
\min _{z \in Y} F\left(\tilde{y}_{k}\right)^{t}\left(z-\tilde{y}_{k}\right) .
$$

We use the CPLEX software to solve this linear problem; given the dimension of the $Y$ 's under consideration — up to a few hundred variables—, this step is very rapid.

- Cuts removal After the addition of several dozens or hundreds of cuts, it is possible to remove many of them without hindering the convergence of the algorithm while improving its speed. The approach of removing cuts with the greatest slacks can slightly improve the speed of the algorithm although convergence cannot be guaranteed. In the experiments reported here, we keep all cuts.

\section{Numerical Results}

We report here on our computational experience with the MATLAB implementation of the algorithm. We include examples both with and without equality constraints. Most problems, except the larger ones, are solved to the precision level $g(y)>-10^{-4}$, evaluated 
at the convex combination points (27); this is a stringent criterion which yields very good accuracies $\left\|y_{k}-y^{*}\right\|$. In most examples, initial box constraints must be set before the first iterations. The variables of all the problems happen to be bounded below by 0 ; the upper bounds in the problems without equalities were set at $1 \times 10^{3}$, and at $1 \times 10^{2}$ for those with equality constraints. This choice has to do with the magnitude of the solution, not with the presence of equality constraints. Some problems are inherently bounded upwards.

Concerning the four options of section 3.1, we proceed as follows. We tested the algorithm using option 2 , both with a tight $(\eta<0.08)$ and relaxed $(\eta<0.9)$ centering. We also tested option 3: with a relaxed centering $(\eta<0.9)$ and a linesearch on the update step, and with a tight centering $(\eta<0.08)$ but no linesearch. Among these four methods, the safest is option 3 with tight centering, which is guaranteed to converge. The results with option 1 were always very similar but slightly worse than to those with option 2 ; we therefore do not report on those tests. We did not try option 4 . Finally, on the large and difficult mmmr problem, section 5.1, we only tested option 2 (for both tight and relaxed centerings).

Results are reported as follows: Center precision refers to the value of $\eta=\|X s-e\|$ at which a point is considered centered. The \# iterations is the number of iterations required to meet the primal gap stopping criterion; it is therefore also the number of analytic centers, and of cutting planes, generated for the problem. The \# of centering steps is the total number of centering steps that were required; this excludes the first step at each iteration, i.e. those steps that we called update steps. As a rule, either one or no centering step at all is required at each iteration. The $c p u$ column indicates the seconds or minutes of cpu time that were required for the completion of the algorithm. All runs except one were performed on an UltraSparc 1 workstation, model 200E, $128 \mathrm{Mb}$ of memory. The $\mathrm{CO}_{2}$ environmental model was run on the multiprocessor HP machine of the E.T.H. in Zürich.

\subsection{An environmental model for pricing $\mathrm{CO}_{2}$ emission permits}

This recent extension of the well-known MARKAL model was developed by a group of researchers at the ETH and the Paul Scherrer Institute in Zürich (see [1] and [3]) Their model, called MMMR for Markal Macro Multi-Regional, integrates the energy models of three countries to allow the possibility of the trade of pollution emission permits; a country can thus buy a right to pollute, while the selling country must reduce its pollution by the same amount.

The MMMR model has two levels. At the international level, the model is a $\operatorname{VI}(\mathcal{F}, Y)$ in ten variables and one equality constraint; this VI represents economic equilibrium conditions. The mapping $\mathcal{F}$ is the (international) excess supply of different goods, including pollution certificates, and is a function of $y$, the prices of the goods. At the national level, a large MARKAL-MACRO model (nonlinear objective, linear constraints) is solved for each country. The national level NLPs take the prices $y$ as parameters, and return the (national) excess supply amounts. The link between the two levels is that the international excess supply is the sum of the national excess supplies. That is, $\mathcal{F}(y)$ is evaluated by solving each of the national level NLPs and adding the national excess supplies achieved by the national solutions. The equality constraint represents a scaling of the prices $y$. One cannot expect the NLPs to have unique solutions, and therefore $\mathcal{F}$ may be a point-to-set mapping, ruling out the existence of the Jacobian of $\mathcal{F}$. Also, the experiments of [1] show that the 
Table 1. Example mmmr dimension $=10$, with option 2

\begin{tabular}{cccc}
\hline Center precision & \# iterations & \# of centering steps & gap \\
\hline 0.1 & 100 & 101 & $-1.2 \mathrm{e}-2$ \\
\hline 0.9 & 106 & 0 & $-1.0 \mathrm{e}-2$ \\
\hline
\end{tabular}

mapping $F$ is "rather" monotone on $Y$, and "almost" pseudo-monotone (in the sense that pseudo-monotonicity holds between most pairs of points and monotonicity between many of them). A further challenge in solving the variational inequality comes from the fact that simply evaluating $\mathcal{F}$ at a certain point takes several minutes on a fast computer, because of the large size of the national level NLPs.

We do not report cpu times for this example: 100 iterations can take 10 hours and more, but spent almost exclusively on the national level NLPs. The 10-dimensional VI itself, excluding the function evaluation, is solved in 0.01 second or less per iteration. Results are shown in Table 1.

\subsection{A Walrasian Equilibrium problem: scarf}

A Walrasian or General Equilibrium is attained when there are no goods whose demand exceeds the supply. Scarf ([39]) has described an economy with a number of goods, consumers and producers, that are related through prices variables and production activities variables.

With the definitions

$\begin{array}{ll}p & \text { prices of the goods }(m \text { by } 1) \\ y & \text { activities levels }(n \text { by } 1) \\ d(p) & \text { consumers demand functions }(m \text { by } 1) \\ A & \text { constant technology or activity matrix; translates } \\ & \begin{array}{l}\text { activity levels into outputs }(m \text { by } n) \\ \text { initial endowment of goods }(m \text { by } 1)\end{array}\end{array}$

the equilibrium is determined by the Nonlinear Complementarity Problem (see [39] and [32] for example)

$$
\begin{array}{ccc}
A^{t} p \leq 0 & y \geq 0 & y^{t} A^{t} p=0 \\
b-d(p)+A y \geq 0 & p \geq 0 & p^{t}(b-d(p)+A y)=0
\end{array}
$$

This formulation, which includes both primal and dual conditions on top of the complementarity, is necessary to apply NCP algorithms. It also hides the equivalent $\operatorname{VI}(F, Y)$ with

$$
F(p)=b-d(p), \quad Y=\{p \mid A p \leq 0, p \geq 0\} .
$$

This primal-only formulation involves $m$ variables instead of the NCP's $m+n$.

In [39], two Walrasian equilibrium examples are given, the largest one with 14 goods and 26 production activities. The resulting NCP has 40 variables, but the equivalent VI only 14 ; the mapping $d(p)$ is of the form $d_{i}(p)=\left(v_{i}^{t} p\right) / p_{i}$, where $v_{i}$ is a constant vector. 
Table 2. Example scarf dimension $=14$

\begin{tabular}{ccccc}
\hline Option & Center precision & \# iterations & \# of centering steps & cpu secs \\
\hline 2 & 0.9 & 104 & 2 & 2.9 \\
\hline 2 & 0.08 & 101 & 101 & 4.0 \\
\hline 3, with search & 0.9 & 102 & 78 & 5.3 \\
\hline 3, no search & 0.08 & 100 & 200 & 4.65 \\
\hline
\end{tabular}

Table 3. Progression in scarf (option 2, center precision 0.9)

\begin{tabular}{cclllll}
\hline \# iterations & gap & $y_{1}$ & $y_{2}$ & $y_{12}$ & $y_{13}$ & $y_{14}$ \\
\hline 1 & -308 & 0.0217 & 0.0239 & 0.5665 & 0.0363 & 0.0304 \\
\hline 50 & -0.1429 & 0.0617 & 0.0583 & 0.0629 & 0.0361 & 0.0930 \\
\hline 103 & $-1.0792 \mathrm{e}-04$ & 0.062144 & 0.058334 & 0.062012 & 0.036515 & 0.092786 \\
\hline 104 & $-9.0878 \mathrm{e}-05$ & 0.062145 & 0.058334 & 0.062012 & 0.036515 & 0.092786 \\
\hline
\end{tabular}

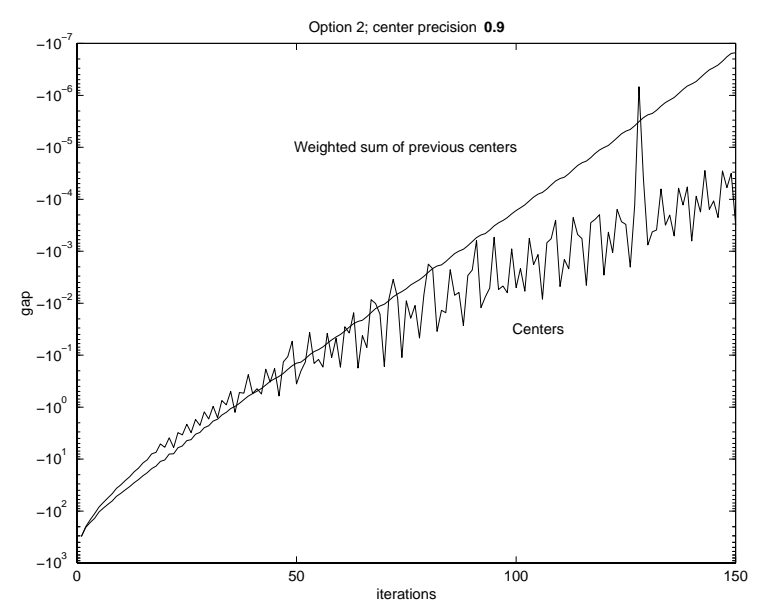

Figure 1. Example scarf: Gap vs iterations

Numerical results are given in Tables 2 and 3. Figure 1 shows the evolution of the gap with respect to the iterations. We display both the gap associated to the analytic centers, and the gap at the weighted average of past centers.

\subsection{Problems from the projection-method literature}

Recently, Solodov and Tseng [40] presented a projection-type method for $V I(F, Y)$ which shares two features with the extragradient method ([24]): the mapping needs not be differentiable, as no Jacobian is used, and in fact needs only be monotone and continuous. These two projection-type methods have a special interest to us, because they tackle the same class 
Table 4. Example qHPHard dimension $=20$

\begin{tabular}{ccccc}
\hline Option & Center precision & \# iterations & \# of centering steps & et ime secs \\
\hline 2 & 0.9 & 220 & 0 & 6.6 \\
\hline 2 & 0.08 & 214 & 218 & 8.3 \\
\hline 3 with search & 0.9 & 214 & 49 & 13 \\
\hline 3 no search & 0.08 & 213 & 426 & 10 \\
\hline
\end{tabular}

Table 5. Example Nash 10 dimension $=10$

\begin{tabular}{ccccc}
\hline Option & Center precision & \# iterations & \# of centering steps & etime secs \\
\hline \hline 2 & 0.9 & 76 & 0 & 1.9 \\
\hline 2 & 0.08 & 69 & 74 & 2.2 \\
\hline 3 with search & 0.9 & 70 & 22 & 2.6 \\
\hline 3 no search & 0.08 & 70 & 138 & 2.4 \\
\hline
\end{tabular}

of problems for which our algorithm is devised: low-monotonicity, no Jacobian, general $Y$. We tested two problems reported in [40], that are the two largest ones without special structure (the authors also solved linear programs and linear complementarity problems with their method). The first problem, called qHPhard, is custom-made and its mapping is the sum of a linear term and a term $\max \left\{0, y_{i}\right\}^{2}$; it has 20 variables. The second problem, called Nash10, is a 10-variable oligopoly problem with a nonlinear mapping, from [15]. In both cases, the set $Y$ used is $\left\{y \in \mathbf{R}_{+}^{m} \mid y_{1}+y_{2}+\ldots+y_{m}=m\right\}$. We report our results in Tables 4 and 5. For the sake of comparison with the results of [40], we report here the elapsed time (MATLAB function et ime), and we used the stopping criterion from [40], which is based on projections. With their method and for, respectively, qHPHard and Nash10, Solodov and Tseng report 555 function evaluations and 251.6 seconds, and 192 function evaluations and 10.6 seconds. They also tested the extragradient method; on these problems, it is $25 \%$ to $50 \%$ slower than their own method, but requires $10 \%$ to $15 \%$ less function evaluations.

We report our results in Tables 4 and 5, and in Figure 2, below.

\subsection{An option pricing model in finance}

In the area of finance, the pricing of stock options and other derivative products has become of paramount importance in the last two decades.

In [19], the authors reformulated the Black-Scholes model for American type options as an infinite-dimensional VI which, through discretization by finite-differences, is approximated by a finite-dimensional VI. For a simple American option with one underlying asset, the discretization takes place along two axes, the current price of the asset and the time to expire. A time-stepping approach allows the problem to be solved as a sequence of VIs, one for each time step, all with the same dimension. We solved a problem with the data:

- Strike price: $K=\$ 10$

- Time span: $T=6$ intervals of 2 weeks

- Price span: $[\$ 0, \$ 20]$ in intervals of $\$ 0.05$. 


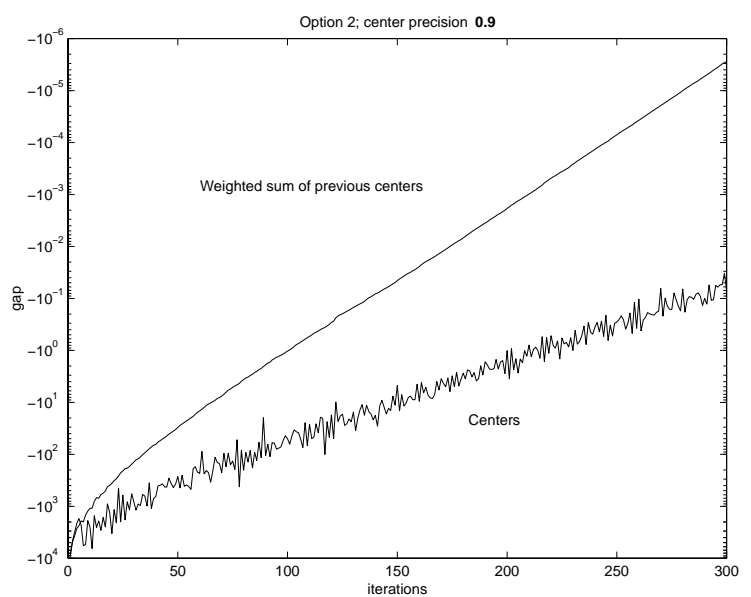

Figure 2. Example qHPHard: Gap vs iterations

Table 6. Example option dimension $=400$

\begin{tabular}{ccccc}
\hline Option & Center precision & \# iterations & \# of centering steps & cpu mins \\
\hline 2 & 0.9 & 1304 & 0 & 170 \\
\hline 2 & 0.08 & 1318 & 1339 & 340 \\
\hline 3 with search & 0.9 & 1272 & 225 & 305 \\
\hline 3 no search & 0.08 & 1317 & 2634 & 542 \\
\hline
\end{tabular}

- Risk-free interest rate: $r=3 \%$

- Diffusion term: $\sigma=0.2$

The resulting sequence of problems $V I(F, Y)$ have 400 variables, and have no other initial constraints than boxes. The mapping $F$ is linear with a non-symmetric Jacobian. This problem is highly structured and sparse while its size grows very rapidly with finer discretizations; in a sense, it is very simple and very large. Our purpose here was not to compete with more specifically-adapted algorithms, but to show that our algorithm can solve problems with a few hundred variables. We were here only interested in solving a "real-world" large VI, so that we report only on the first of the six 400-variable problems. For more details on the model, see [44] or the appendix of the thesis [6]. Huang and Pang [18] discuss the pricing of options with LCP (Linear Complementarity Problem) algorithms which take advantage of the inherent linearity and sparsity of this application.

Numerical results are shown in Tables 6 and 7. Given the size of the problem, we stopped the algorithm when it reached a gap $>-10^{-3}$. Note that because of boundary conditions, the values at either end of the solution vector in $\mathbf{R}^{400}$, i.e. $y_{1}^{*}, y_{2}^{*}, \ldots$ and $\ldots, y_{399}^{*}, y_{400}^{*}$ are known in advance with rather good precision. Also, the units in Table 7 are dollars, so one observes that already at iteration 1000 , the accuracy is within a tenth of a cent. 
Table 7. Progression in option

\begin{tabular}{cccccccc}
\hline \# iterations & gap & $y_{1}$ & $y_{2}$ & $y_{199}$ & $y_{200}$ & $y_{399}$ & $y_{400}$ \\
\hline 1 & -885 & 10.0500 & 10.0125 & 2.6250 & 2.5875 & 0.0750 & 0.0625 \\
\hline 500 & -0.999 & 10.0316 & 9.9844 & 0.2387 & 0.2229 & 0.0223 & 0.0170 \\
\hline 1000 & -0.0088 & 10.0018 & 9.9518 & 0.2163 & 0.2018 & 0.0021 & 0.0016 \\
\hline 1303 & -0.0010 & 10.0003 & 9.9503 & 0.2159 & 0.2014 & 0.0006 & 0.0005 \\
\hline 1304 & -0.00099 & 10.0003 & 9.9503 & 0.2159 & 0.2014 & 0.0006 & 0.0005 \\
\hline
\end{tabular}

Table 8. Example choi dimension $=13$

\begin{tabular}{ccccc}
\hline Option & Center precision & \# iterations & \# of centering steps & cpu secs \\
\hline 2 & 0.9 & 67 & 0 & 13 \\
\hline 2 & 0.08 & 66 & 70 & 13 \\
\hline 3 with search & 0.9 & 62 & 18 & 13.2 \\
\hline 3 no search & 0.08 & 63 & 124 & 13.6 \\
\hline
\end{tabular}

\subsection{Other problems without linear equality constraints}

The problems in this section do not use the capability of the algorithm to handle equality constraints.

The first example is a Nash equilibrium, the second one a spatial price equilibrium problem, the third is a traffic assignment problem and the fourth is an energy model. They are available from MCPLIB [7], and more information on them is available in the specific references as well as in [7].

- Example choi: Choi, DeSarbo and Harker [4] have used a Nash equilibrium in a model for the pricing of analgesics. With $p$ the vector of prices of 13 analgesics, the Nash equilibrium of this application is a solution of the $V I(F, Y)$; here $F(p)$ is a vector with components $-d \Pi_{j}(p) / d p_{j}$ and $\Pi_{j}(p)$ is the profit function of the $j^{\text {th }}$ firm. The profit function $\Pi_{j}(p)$ is considered to be a function of $p_{j}$ only, the other prices remaining constant.

In the instance described in [4], the mapping $F$ is nonlinear and is not a gradient mapping, i.e. its Jacobian is not symmetric. The results are shown in Table 8 and in Figure 3.

- Example tobin: The second problem is a well-known spatial price equilibrium model from Tobin [43]. It is a so-called price formulation: supply and demand at the different markets are functions of the prices. In opposition to the quantity formulation, this one involves no equality constraints. This model has two interacting commodities on five markets or regions. The transportation cost, demand and supply functions are nonlinear, and the problem is asymmetric, as well as non-monotone at the solution. See Tables 9 and 10 below. Figure 4 shows, for two levels of recentering precision, the evolution of the gap with respect to the iterations. We display both the gap associated to the analytic centers, and the gap at the weighted average of past centers. No satisfactory explanation could be found for the kink around 720 iterations.

- Example gafni and bertsekas: This interesting application to traffic assignment was described in [2]. There are two strictly equivalent formulations of the same problem 


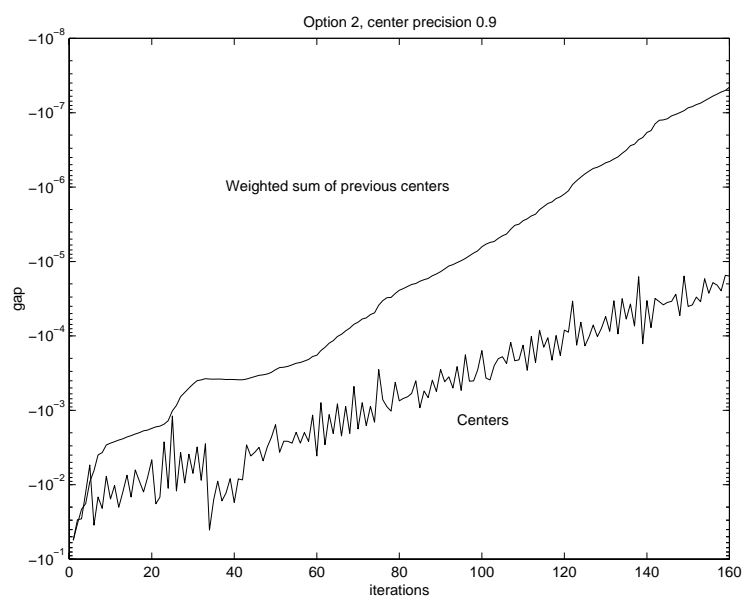

Figure 3. Example choi: Gap vs iterations

Table 9. Example tobin dimension $=42$

\begin{tabular}{ccccc}
\hline Option & Center precision & \# iterations & \# of centering steps & cpu secs \\
\hline 2 & 0.9 & 703 & 0 & 47 \\
\hline 2 & 0.08 & 688 & 692 & 75 \\
\hline 3 with search & 0.9 & 697 & 125 & 218 \\
\hline 3 no search & 0.08 & 687 & 1368 & 163 \\
\hline
\end{tabular}

Table 10. Progression in tobin (option 2, center precision 0.9)

\begin{tabular}{cccccccc}
\hline$\#$ iterations & gap & $y_{1}$ & $y_{9}$ & $y_{17}$ & $y_{40}$ & $y_{41}$ & $y_{42}$ \\
\hline 1 & $-1 \mathrm{e} 12$ & 500 & 500 & 500 & 500 & 500 & 500 \\
\hline 250 & -113.1288 & 0.0689 & 3.9642 & 12.7263 & 22.7554 & 18.7534 & 29.1753 \\
\hline 500 & -0.0994 & $3.7882 \mathrm{e}-05$ & 3.8827 & 12.7678 & 22.7644 & 18.7604 & 29.1615 \\
\hline 702 & -0.00011 & $7.1856 \mathrm{e}-08$ & 3.8827 & 12.7679 & 22.7644 & 18.7604 & 29.1615 \\
\hline 703 & $-0.81 \mathrm{e}-05$ & $6.99 \mathrm{e}-08$ & 3.8827 & 12.7679 & 22.7644 & 18.7604 & 29.1615 \\
\hline
\end{tabular}

Table 11. Example bert sekas dimension $=15$

\begin{tabular}{ccccc}
\hline Option & Center precision & \# iterations & \# of centering steps & cpu secs \\
\hline 2 & 0.9 & 197 & 0 & 4.9 \\
\hline 2 & 0.08 & 194 & 193 & 7.3 \\
\hline 3 with search & 0.9 & 191 & 36 & 11.8 \\
\hline 3 no search & 0.08 & 195 & 338 & 11.5 \\
\hline
\end{tabular}

(see the results in Tables 11 and 12). The first formulation is a nonlinear complementarity problem (NCP) in 15 variables while the second is a more general mixed complementarity problem (MCP) in 5 variables. The delay functions are quadratic, and the nonsymmetry of the problem is controlled through a parameter $\gamma$. While both formulations are just specialized variational inequality problems and can thus be solved as VIs, the lower dimension of the MCP is very favorable to the speed of the algorithm. 


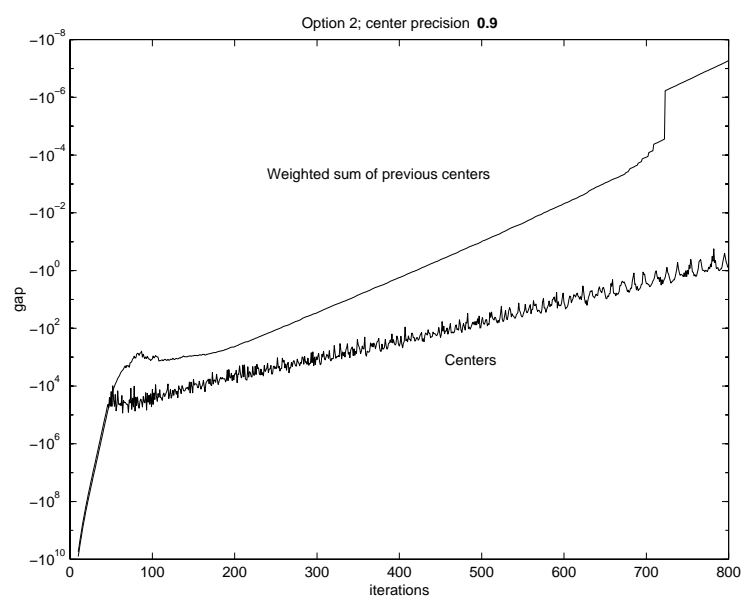

Figure 4. Example tobin: Gap vs iterations

Table 12. Example gafni dimension $=5$

\begin{tabular}{ccccc}
\hline Option & Center precision & \# iterations & \# of centering steps & cpu secs \\
\hline 2 & 0.9 & 43 & 0 & 0.95 \\
\hline 2 & 0.08 & 41 & 41 & 1.4 \\
\hline 3 with search & 0.9 & 41 & 13 & 1.8 \\
\hline 3 no search & 0.08 & 41 & 79 & 1.9 \\
\hline
\end{tabular}

Table 13. Example et amge dimension $=114$

\begin{tabular}{ccccc}
\hline Option & Center precision & \# iterations & \# of centering steps & cpu minutes \\
\hline 2 & 0.9 & 2980 & 7 & 82 \\
\hline 2 & 0.08 & 3487 & 3137 & 199 \\
\hline 3 with search & 0.9 & 3255 & 348 & 399 \\
\hline 3 no search & 0.08 & 3186 & 6370 & 376 \\
\hline
\end{tabular}

- Example et amge: This is a general equilibrium model of A. Manne [30]. The model represents the interactions between the energy sector and the economy of the United States and comprises 114 variables. See the numerical results in Table 13 below.

\subsection{Other problems with linear equality constraints}

We present computational experience with two spatial price equilibrium problems that are readily available in the literature. The so-called quantity formulation of such problems uses inverse demand and supply functions, i.e. price functions in terms of the quantities. With this formulation the equilibrium is defined as a variational inequality problem with (linear) conservation of flow constraints; the general format is: 
Table 14. Example nagurney dimension $=8$

\begin{tabular}{ccccc}
\hline Option & Center precision & \# iterations & \# of centering steps & cpu secs \\
\hline 2 & 0.9 & 61 & 0 & 1.2 \\
\hline 2 & 0.08 & 55 & 59 & 1.7 \\
\hline 3 with search & 0.9 & 55 & 19 & 1.9 \\
\hline 3 no search & 0.08 & 56 & 111 & 1.9 \\
\hline
\end{tabular}

Table 15. Example harker dimension $=26 \gamma=1$

\begin{tabular}{ccccc}
\hline Option & Center precision & \# iterations & \# of centering steps & cpu secs \\
\hline 2 & 0.9 & 293 & 0 & 15 \\
\hline 2 & 0.08 & 282 & 283 & 19 \\
\hline 3 with search & 0.9 & 288 & 67 & 30 \\
\hline 3 no search & 0.08 & 283 & 566 & 24 \\
\hline
\end{tabular}

$$
\left[\pi\left(s^{*}\right), c\left(T^{*}\right),-\rho\left(d^{*}\right)\right] \cdot\left[\begin{array}{c}
s-s^{*} \\
T-T^{*} \\
d-d^{*}
\end{array}\right] \geq 0, \quad \forall(s, T, d) \in K
$$

where $s, T$ and $d$ are respectively the supply, shipments, and demand quantities, $\pi, c$ and $\rho$ are supply, demand, and shipment prices, and $K=\left\{(s, T, d) \mid s_{i}=\sum_{j=1}^{n} T_{i j}, d_{j}=\sum_{i=1}^{m} T_{i j}\right\}$ ensures flow conservation.

For a general reference, see [35]. Our algorithm allows us to work directly with this original formulation.

- Example nagurney: Our first example is a small problem from A. Nagurney's book [35]. It has 2 supply markets and 2 demand markets; with the shipment quantities on the 4 arcs, it has dimension 8 . The transportation costs, supply prices and demand prices are all linear functions. The Jacobian of $F$ in the $V I(F, Y)$ formulation is asymmetric, so that no equivalent convex optimization formulation exists.

The results are given in Table 14.

- Example harker: The second equality-constrained example (see [15]) has 5 markets all acting as demand and supply markets for the traded commodity. There are 16 connecting arcs for a total of 26 variables. All functions are polynomial and nonlinear. This problem includes a parameter $\gamma$ which controls the level of asymmetry of the Jacobian of $F$, with $\gamma=0$ implying a symmetric matrix. Results are shown for two values of $\gamma, 1$ and 15; see Tables 15 and 16. It is especially interesting to note the influence of asymmetry. Symmetrized methods such as the ones discussed in [15] are, quite naturally, sensitive to deviations from symmetry: there the $\gamma=15$ case takes twice the time and the iterations of the $\gamma=1$ case ( 4 minutes vs 2 minutes, on a pre-1988 computer). Our algorithm seems on the other hand to be unaffected by asymmetry.

\section{Conclusion}

We have presented an algorithm for pseudomonotone or monotone variational inequalities $V I(\mathcal{F}, Y)$ over convex sets. At each iteration, it requires one function evaluation to generate 
Table 16. Example harker dimension $=26 \gamma=15$

\begin{tabular}{ccccc}
\hline Option & Center precision & \# iterations & \# of centering steps & cpu secs \\
\hline 2 & 0.9 & 265 & 0 & 13 \\
\hline 2 & 0.08 & 261 & 261 & 17 \\
\hline 3 with search & 0.9 & 272 & 70 & 27 \\
\hline 3 no search & 0.08 & 261 & 522 & 22 \\
\hline
\end{tabular}

a cut, and a second one if the gap at the weighted average point is to be computed. Beyond that, each iteration basically consists of solving from one to four linear systems, depending on the desired recentering precision (which controls the number of centering steps) and the presence or not of equality constraints. The mapping $\mathcal{F}$ need not be differentiable and may be multivalued. The set $Y$ can be defined by linear equalities and inequalities, or implicitly by a separation oracle.

Our approach may not be competitive with specialized methods for problems with special features: LCP's, differentiable NCP's and MCP's, strongly monotone and differentiable VI's, etc. However, when the Jacobian is difficult to obtain, or simply not defined (e.g. in multi-valued cases), it is our opinion that there are few other efficient alternatives. Furthermore, the low level of monotonicity required in practice (pseudo-monotonicity or plain monotonicity) makes the ACCPM-VI method with linear cuts attractive both in terms of robustness and speed. This is well illustrated in the MMMR application above.

The update from one center to the next is done very efficiently using option 2: recentering steps are almost never needed if a loose centering condition is used, and this justifies the use of a centering term in the updating step. This shows that a primal-dual approach is a little bit more efficient than a pure primal or a pure dual one.

\section{Acknowledgments}

We want to express our gratitude to B. Büeler and H.-J. Lüthi of the E.T.H. Zürich for making both their MMMR model and their computer available to us for testing. We also thank J. Detemple, P. Marcotte, Y. Nesterov and J.-Ph. Vial for stimulating discussions and helpful comments. The very careful analysis by two anonymous referees significantly improved the presentation of this paper.

\section{Notes}

1. centering steps are discussed later

2. Pseudo-co-coercive with modulus $\alpha$ is defined as $F\left(y_{1}\right)^{t}\left(y_{2}-y_{1}\right) \Rightarrow F\left(y_{2}\right)^{t}\left(y_{2}-y_{1}\right) \geq \alpha \| F\left(y_{1}\right)-$ $F\left(y_{2}\right) \|^{2}$ for all $y_{1}, y_{2} \in Y$.

3. The notation $O^{*}$ indicates that lower order terms are ignored

4. A formal proof of this would require a very significant and lengthy rewriting of the proofs of [12] and [13].

\section{References}

1. O. Bahn, B. Büeler, S. Kypreos and H.-J. Luethi, "Modeling an international market of CO2 emission permits," International Journal Global Energy Issues, submitted 1997. 
2. D. Bertsekas and E. Gafni, "Projection Methods for variational inequalities with applications to the traffic assignment problem," Mathematical Programming, vol. 17, pp. 139-159, 1982.

3. B. Büeler, "Computing Economic Equilibria and its Application to International Trade of $\mathrm{CO}_{2}$ Permits: An Agent-Based Approach," Ph.D. thesis, Swiss Federal Institute of Technology, Zürich, Switzerland, December 1997.

4. S. Choi, W. DeSarbo and P. Harke, "Product positioning under price competitition," Management Science, vol. 36-2, pp. 175-199, 1990.

5. J.-P. Crouzeix, "Pseudomontone variational inequality problems: Existence of solutions," Mathematical Programming, vol. 78, pp. 305-314, 1997.

6. M. Denault, "Variational Inequalities with Analytic Center Cutting-Plane Methods," Ph.D. thesis, McGill University, Montréal, Canada, 1998.

7. S. Dirkse and M. Ferris, "MCPLIB: A collection of nonlinear mixed complementarity problems," Optimization Methods and Software, vol. 5, pp. 319-345, 1995.

8. F. Facchinei, A. Fischer and C. Kanzow, "A semismooth Newton method for variational inequalities: the case of box constraints," in Complementarity and variational problems: State of the art, Society for Industrial and Applied Mathematics (SIAM), Philadelphia, 1997.

9. M. Ferris and J.-S. Pang, "Complementarity and variational problems: State of the art," Society for Industrial and Applied Mathematics (SIAM), Philadelphia, 1997.

10. M. Fukushima, "Equivalent differentiable optimization problems and descent methods for asymmetric variational inequality problems," Mathematical Programming, vol. 53, pp. 99-110, 1992.

11. J.-L. Goffin, A. Haurie and J.-P. Vial, "Decomposition and nondifferentiable optimization with the projective algorithm," Management Science, vol. 38, pp. 284-302, 1992.

12. J.-L. Goffin, Z.-Q. Luo and Y. Ye, "Complexity Analysis of an Interior Cutting Plane Method for Convex Feasibility Problems," SIAM Journal on Optimization, vol 6, pp. 638-652, 1996.

13. J.-L. Goffin, P. Marcotte and D. Zhu, "An analytic center cutting plane method for pseudomonotone variational inequalities," Operations Research Letters, vol. 20, pp. 1-6, 1997.

14. J.-L. Goffin and F. Mokhtarian, "Using the primal-dual infeasible Newton method in the analytic center method for problems defined by deep cutting planes," Journal of Optimization Theory and Applications, vol. 37, pp. 181-210, 1998.

15. P. Harker, "Accelerating the convergence of the diagonalization and projection algorithms for finitedimensional variational inequalities," Mathematical Programming, vol. 41, pp. 29-59, 1988.

16. P. Harker, "Lectures on Computation of Equilibria with Equation-Based Methods,", CORE Lecture Series, CORE Foundation, Louvain-la-Neuve, 1993.

17. P. Harker and J.-S. Pang, "Finite-dimensional variational inequality and nonlinear complementarity problems: a survey of theory, algorithms and applications," Mathematical Programming, vol. 48 (1990), 161-220, 1990.

18. J. Huang and J.-S. Pang, "Option Pricing and Linear Complementarity," Journal of Computational Finance, submitted 1997.

19. P. Jaillet, D. Lamberton, and B. Lapeyre, "Variation Inequalities and the Pricing of American Options," Acta Applicandae Mathematicae, vol. 21, pp. 263-289, 1990

20. C. Kanzow and H.-D. Qi, "A QP-free constrained Newton-type method for variational inequality problems," Preprint 121, Institute of Applied Mathematics, University of Hamburg, Hamburg, Germany, 1997.

21. S. Karamardian, "Complementarity problems over cones with monotone and pseudomonotone maps," Journal of Optimization Theory and Applications, vol. 18, pp. 445-454, 1976.

22. D. Kinderlehrer and G. Stampacchia, "An Introduction to Variational Inequalities and their applications," Academic Press, New York, 1980.

23. I. V. Konnov, "A combined relaxation method for variational inequalties with nonlinear constraints," Mathematical Programming, vol. 80, pp. 239-252, 1998.

24. G. M. Korpelevich, "The extragradient method for finding saddle points and other problems," Matecon, vol. 12, pp. 747-756, 1976

25. C. Lemaréchal, A. Nemirovskii and Y. Nesterov, "New Variants of Bundle Methods, "Mathematical Programming, vol. 69, pp.111-147, 1995.

26. H.-J. Lüthi, "On the solution of variational inequalities by the ellipsoid method," Mathematics of Operations Research, vol. 10-3, pp. 515-522, 1985.

27. T. Magnanti and G. Perakis, "A unifying geometric solution framework and complexity analysis for variational inequalities," Mathematical Programming, vol. 71, pp. 327-351, 1995. 
28. T. Magnanti and G. Perakis, "Averaging schemes for variational inequalities and systems of equations," Mathematics of Operations Research, pp. 3-22, pp. 568-587, 1997.

29. O. Mangasarian, "Equivalence of the complementarity problem to a system of nonlinear equations," SIAM Journal on Applied Mathematics, vol. 31, pp. 89-92, 1976.

30. A.S. Manne, "ETA-MACRO: A Model of Energy- Economy Interactions," in Modeling Energy-Economy Interactions, Resources for the Future, Washington, D.C, 1977.

31. P. Marcotte, "Inéquations variationnelles: Motivation, algorithmes de résolution et quelques applications," Centre de Recherche sur les Transports, Publication CRT-97-02, 1997.

32. L. Mathiesen, "An algorithm based on a sequence of linear complementarity problems applied to a wlarasian equilibrium model: an example," Mathematical Programming, vol 37, pp. 1-18, 1997.

33. G. Minty, "Monotone (nonlinear) operators in Hilber space," Duke Mathematics Journal, vol. 29, pp. 341346,1962

34. S. Mizuno, "A new polynomial time method for a linear complementarity problem," Mathematical Programming, vol. 56, pp. 31-43, 1992

35. A. Nagurney, "Network Economics: a variational inequality approach," Kluwer Academic Publishers: Boston, MA, 1993

36. Y. Nesterov and A. Nemirovskii, "Interior-Point Polynomial Algorithms in Convex Programming," SIAM Studies in Applied Mathematics, SIAM: Philadelphia, 1994.

37. Y. Nesterov and J.-Ph. Vial, "Homogeneous analytic center cutting plane methods for convex problems and variational inequalities," Logilab Technical Report, 1997.4, 1997.

38. D. Ralph and S. Wright, "Superlinear convergence of an interior-point method for monotone variational inequalities," in Complementarity and variational problems: State of the art, Society for Industrial and Applied Mathematics (SIAM): Philadelphia, 1997.

39. H. Scarf (with the collaboration of T. Hansen), "The Computation of Economic Equilibria," Yale University Press, 1973

40. M. Solodov and P. Tseng, "Modified projection-type methods for monotone variational inequalities," SIAM Journal on Control and Optimization, vol. 34-5, pp. 1814-1830, 1996

41. Gy. Sonnevend, "New algorithms in convex programming based on a notion of "centre" (for systems of analytic inequalities) and on rational extrapolation," in Trends in Mathematical Optimization, Birkhäuser Verlag, pp. 311-326, 1988

42. D. Sun, M. Fukushima and L. Qi, "A computable generalized hessian of the D-gap function and Newton-type methods for variational inequality problems," in Complementarity and variational problems: State of the art, Society for Industrial and Applied Mathematics (SIAM): Philadelphia, 1997.

43. R. Tobin, "A variable dimension solution approach for the general spatial price equilibrium problem, "Mathematical Programming, vol. 40, pp. 33-51, 1988

44. P. Wilmott, J.N. Dewynne and S.D. Howison, "Option Pricing: Mathematical Models and Computation," Oxford Financial Press, 1993.

45. B. Xiao and P. Harker, "A nonsmooth Newton method for variational inequalities, I: theory", Mathematical Programming, vol. 65, pp. 151-194, 1994.

46. B. Xiao and P. Harker, "A nonsmooth Newton method for variational inequalities, II: numerical results," Mathematical Programming, vol. 65, 195-216, 1994.

47. Y. Ye, "Interior Point Algorithms: Theory and Analysis," John Wiley and Sons, Inc., 1997.

48. Y. Zhang, "Solving Large-Scale Linear Programs by Interior-Point Methods Under the MATLAB Environment,” Technical Report TR96-01, University of Maryland Baltimore County, 1996. 